\title{
PD-1/PD-L1 expression and tumor-infiltrating lymphocytes are prognostically favorable in advanced high-grade serous ovarian carcinoma
}

\author{
Laura Martin de la Fuente ${ }^{1}$ (D) $\cdot$ Sofia Westbom-Fremer ${ }^{1,2} \cdot$ Nicolai Skovbjerg Arildsen $^{1} \cdot$ Linda Hartman $^{1}$. \\ Susanne Malander ${ }^{1} \cdot$ Päivi Kannisto $^{3} \cdot$ Anna Måsbäck $^{2} \cdot$ Ingrid Hedenfalk $^{1}$
}

Received: 11 October 2019 / Revised: 7 January 2020 / Accepted: 9 January 2020 / Published online: 24 January 2020

(C) The Author(s) 2020

\begin{abstract}
The response rate to checkpoint inhibitors for women with high-grade serous carcinoma of the ovary, fallopian tube, and peritoneum (HGSC) is modest, and development of predictive biomarkers is needed. The main focus has been on tumor cell PD-L1 expression, but its assessment alone is insufficient for patient selection in most malignancies. We mapped the presence of macrophages (CD68 and CD163) and lymphocytes (CD3) located within the tumor epithelium, the cell type-specific expression of PD-L1 and PD-1, and their impact on 5-year overall survival (OS) in a consecutive cohort of 130 women diagnosed with advanced HGSC between 2011 and 2015. PD-L1 was expressed mainly by macrophages (not by tumor cells) and PD-1 by lymphocytes. Women with higher CD3, PD-L1, and PD-1 expression had improved OS $(P=0.03, P=0.007$, and $P=0.02$, respectively). In the external data set (203 women), high expression of CD274 (encoding PD-L1) was associated with improved OS $(P=0.03)$, in accordance with our results. Furthermore, higher CD163 expression was associated with better outcome in women with no residual tumor after primary surgery $(P=0.02)$. Thus, women with greater lymphocyte tumor infiltration had better outcome and PD-L1/PD-1 expression, regardless of PD-1/PD-L1 being markers for immune suppressive pathways, conferred a survival benefit in our cohort. Our results highlight that tumor immunity may be harnessed in subsets of HGSC.
\end{abstract}

Keywords PD-1/PD-L1 pathway $\cdot$ Tumor-infiltrating lymphocytes $\cdot$ Macrophages $\cdot$ Prognostic marker

\section{Introduction}

High-grade serous carcinoma of the ovary, fallopian tube, and peritoneum (HGSC) is the most common and lethal subtype of

Electronic supplementary material The online version of this article (https://doi.org/10.1007/s00428-020-02751-6) contains supplementary material, which is available to authorized users.

Laura Martin de la Fuente

laura.martin_de_la_fuente@med.lu.se

1 Department of Clinical Sciences, Division of Oncology and Pathology, Lund University and Skåne University Hospital, Lund, Sweden

2 Department of Surgical Pathology, Division of Laboratory Medicine, Skåne University Hospital, Lund, Sweden

3 Department of Obstetrics and Gynaecology Lund, Skåne University Hospital, Lund University, Lund, Sweden epithelial ovarian carcinoma [1]. Due to the lack of symptoms, it typically presents at an advanced stage. Cytoreductive surgery is the most important treatment affecting outcome, and patients with no macroscopic residual tumor after primary surgery have a survival benefit [2]. Surgery is followed by platinum-based adjuvant chemotherapy. Despite a majority of women with advanced HGSC initially responding to treatment, many suffer relapses and the cancer cells have then often developed resistance or are less sensitive to chemotherapy. Thus, advanced stage at diagnosis and a high rate of relapses are the main reasons for the poor prognosis of this disease. Although no great improvements in outcome have been made over the past decades, the recent introduction of poly (ADP-ribose) polymerase (PARP) inhibitors into the clinical practice seems promising in transforming survival prospects for women with advanced HGSC [3].

The introduction of checkpoint inhibitor-based antibodies directed at CTLA-4, PD-1, and PD-L1 receptors has improved survival for many cancer patients. In particular, in advanced malignant melanoma, lung cancer and bladder cancer clinical 
trials have shown increased overall survival (OS) and longtime survivors [4-6]. To date, the response rate to checkpoint inhibitors for patients with HGSC seems to be modest [7, 8]. However, there is hope for increased response rates through patient selection and combination of therapies. For example, there is emerging preclinical data suggesting that the patient population that responds to PARP inhibition and PD-1/PD-L1 antibodies may significantly overlap [9], and it is hypothesized that increased DNA damage by PARP inhibition will increase the number of tumor neoantigens, creating a more antigenic environment in which to stimulate the immune microenvironment [10]. Thus, development of predictive biomarkers is needed to identify the subset of patients who will benefit from treatment and to minimize the risk of toxicities. The main focus to date has been on tumor cell PD-L1 expression, but its assessment alone is insufficient for patient selection in most malignancies [11].

The survival advantage of high numbers of tumorinfiltrating lymphocytes in HGSC has been shown in several studies $[12,13]$. Furthermore, global gene expression analyses have identified an immunoreactive molecular subtype [14, 15], and showed its value as a predictor of improved survival compared with the other molecular subtypes [16]. However, the prognostic value of PD-1 and PD-L1 in HGSC has been studied with ambiguous results [17-20]. Mapping the expression of PD-1/PD-L1 and immune cells in HGSC is clinically relevant because in addition to its prognostic value, it may provide important information for further study of their potential to predict treatment response to immunotherapy. Furthermore, a negative effect of CD163+ tumor-associated macrophages on survival was reported in a meta-analysis of patients with ovarian carcinoma [21]. Therefore, we characterized the macrophage population by evaluating CD163 in our cohort, a marker for alternatively activated macrophages (M2) considered to promote tumor progression.

Thus, in this study, we mapped the presence of macrophages and lymphocytes located within the tumor epithelium, the cell type-specific expression of PD-L1 and PD-1 and their impact on prognosis in a well-characterized, contemporary and consecutive cohort of 130 women diagnosed with advanced HGSC.

\section{Methods}

This study followed the REMARK (Reporting Recommendations for Tumor Marker Prognostic Studies) guidelines [22].

\section{Patients}

Ethical approval for this study was granted by the Ethics Committee at Lund University, Sweden, waiving the requirement for informed consent. A total of 156 consecutive cases of HGSC were selected at the Gynecology Department in the southern Swedish healthcare region between 2011 and 2015. All cases were reviewed by a gynecologic pathologist according to the World Health Organization Classification 2014 [23] (excluded patients shown in Supplementary Fig. 1) and staged according to the International Federation of Gynecology and Obstetrics criteria [24]. All tumor samples were collected at primary surgery or diagnostic biopsy prior to chemotherapy administration. Eleven women with stage I and II disease were excluded from the analysis as we were not able to find regression model fitting all stages and due to their remarked difference in prognosis. Thus, 130 women with stage III and IV disease were included in the analysis. With the exception of three patients who underwent bowel obstruction surgery only, all patients underwent primary cytoreductive surgery (two patients delayed primary and the rest upfront primary). The complete resection rate (no macroscopic tumor) for upfront and delayed primary surgery was $75 /$ 127 (59\%). Four cases, where upfront primary cytoreductive surgery failed, were redirected to neoadjuvant chemotherapy, and of the two that could undergo interval surgery, none achieved complete resection. Platinum-based chemotherapy was administered to all but four of the 130 patients, and three patients died after only one chemotherapy cycle had been administered. Of the 123 patients who completed chemotherapy, $87(71 \%)$ were evaluated as complete response, $29(24 \%)$ as partial response, one as stable disease and six as progressive disease, by the end of treatment. Platinum resistance was defined according to the consensus achieved by the Gynecologic Cancer InterGroup (Vancouver, June 2010) [25], and data are presented in Table 1. Sixteen patients with residual tumor after primary surgery received bevacizumab together with the platinum-based regime. Last follow-up date for all patients was December 2018. Median follow-up time for the patients included was 39 months (0.389.0) (events and more detailed survival data in Table 1).

\section{Tissue microarray construction and immunohistochemistry}

Viable tumor areas from multiple sites were selected from formalin-fixed paraffin-embedded tissue blocks: four cores from the adnexa (two different blocks), two cores from lymph node metastases (if present), and two or four cores from peritoneal metastases. Thus, 6-8 1-mm core needle biopsies from multiple sites were available from each patient in most cases (of 130 women, 19 cases had 4 cores, 68 cases 6 cores, and 43 cases 8 cores). Sections, $3-4 \mathrm{~mm}$ in thickness, were deparaffinized, rehydrated, and stained. A summary of antibodies and immunohistochemistry procedures used is provided in Table 2. The sections were incubated with primary antibody (detailed incubation conditions in Table 2). The visualization systems applied were EnVision FLEX (Agilent Dako) for the Dako Autostainer platform and ultraView Universal 
Table 1 PD-1, PD-L1, CD3, CD68, and CD163 expression and clinical parameters

\begin{tabular}{|c|c|c|c|c|c|c|c|c|c|}
\hline & & $N(\%)$ & \multicolumn{2}{|c|}{$\begin{array}{l}\text { PD-1 low } \\
N(\%)\end{array}$} & $\begin{array}{l}\text { PD-1 high } \\
N(\%)\end{array}$ & $P$ value & $\begin{array}{l}\text { PD-L1 low } \\
N(\%)\end{array}$ & $\begin{array}{l}\text { PD-L1 high } \\
N(\%)\end{array}$ & $P$ value \\
\hline \multicolumn{2}{|l|}{ Advanced HGSC } & 130 & \multicolumn{2}{|l|}{91} & 39 & & 104 & 26 & \\
\hline \multicolumn{10}{|l|}{ Age } \\
\hline Mean & & 67 & \multicolumn{2}{|l|}{68} & 65 & \multirow[t]{2}{*}{$0.5^{\mathrm{c}}$} & 68 & 63 & \multirow[t]{2}{*}{$0.2^{\mathrm{c}}$} \\
\hline Range & & $43-86$ & $43-86$ & & $45-85$ & & $43-86$ & $51-85$ & \\
\hline \multicolumn{10}{|l|}{ Residual tumor } \\
\hline No & & $75(58)$ & \multicolumn{2}{|l|}{$50(55)$} & $25(64)$ & \multirow[t]{2}{*}{$0.3^{\mathrm{d}}$} & $57(55)$ & $18(69)$ & \multirow[t]{2}{*}{$0.2^{\mathrm{d}}$} \\
\hline Yes & & $55(42)$ & $41(45)$ & & $14(36)$ & & $47(45)$ & $8(31)$ & \\
\hline \multicolumn{10}{|l|}{ Stage } \\
\hline III & & 99 (76) & \multicolumn{2}{|l|}{$67(74)$} & $32(82)$ & \multirow[t]{2}{*}{$0.3^{\mathrm{d}}$} & $77(74)$ & $22(85)$ & \multirow[t]{2}{*}{$0.3^{\mathrm{d}}$} \\
\hline IV & & $31(24)$ & $24(26)$ & & $7(18)$ & & $27(26)$ & $4(15)$ & \\
\hline \multicolumn{10}{|l|}{ PFI } \\
\hline 12 months & & $64(52)$ & \multicolumn{2}{|l|}{$37(44)$} & $27(71)$ & \multirow[t]{4}{*}{$0.009^{e}$} & $49(50)$ & $15(60)$ & \multirow[t]{4}{*}{$0.3^{\mathrm{e}}$} \\
\hline $6-12$ months & & $29(24)$ & $25(29)$ & & $4(11)$ & & $24(25)$ & $5(20)$ & \\
\hline$<6$ months & & $30(24)$ & \multicolumn{2}{|l|}{$23(27)$} & $7(18)$ & & $25(25)$ & $5(20)$ & \\
\hline No platinum & & 7 & & & & & & & \\
\hline \multicolumn{10}{|l|}{ 5-Year OS } \\
\hline Events/person years & & $85^{\mathrm{b}} / 388$ & \multicolumn{2}{|l|}{$67 / 253$} & $18 / 135$ & & $74 / 298$ & $11 / 90$ & \\
\hline 5-Year OS $(\%)$ & & 29.5 & 21.7 & & 49.3 & & 23.6 & 56.4 & \\
\hline 5-Year PFS & & & & & & & & & \\
\hline Events/person years & & $107 / 257$ & $82 / 155$ & & $25 / 102$ & & $89 / 196$ & $18 / 61$ & \\
\hline 5-Year PFS (\%) & & 16.5 & 9.4 & & 33 & & 14 & 26 & \\
\hline & $\mathrm{CD} 3$ low & CD3 high & $P$ value & CD68 low & CD68 high & $P$ value & CD163 low & CD163 high & $P$ value \\
\hline & $N(\%)$ & $N(\%)$ & & $N(\%)$ & $N(\%)$ & & $N(\%)$ & $N(\%)$ & \\
\hline Advanced HGSC & 87 & 43 & & 60 & 70 & & 79 & 51 & \\
\hline Age & & & & & & & & & \\
\hline Median & 68 & 65 & $0.08^{\mathrm{c}}$ & 68 & 65 & $0.03^{c}$ & 68 & 66 & $0.1^{\mathrm{c}}$ \\
\hline Range & $43-86$ & $45-85$ & & $45-86$ & $45-85$ & & $45-86$ & $43-80$ & \\
\hline Residual tumor & & & & & & & & & \\
\hline No & $52(60)$ & $23(53)$ & $0.5^{\mathrm{d}}$ & $38(63)$ & $37(53)$ & $0.2^{\mathrm{d}}$ & $47(49.5)$ & $28(55)$ & $0.6^{\mathrm{d}}$ \\
\hline Yes & $35(40)$ & $20(47)$ & & $22(37)$ & $33(47)$ & & $32(40.5)$ & $23(45)$ & \\
\hline Stage & & & & & & & & & \\
\hline III & $65(75)$ & $34(79)$ & $0.6^{\mathrm{d}}$ & $47(78)$ & $52(74)$ & $0.6^{\mathrm{d}}$ & $60(76)$ & $39(76.5)$ & $0.9^{\mathrm{d}}$ \\
\hline IV & $22(25)$ & $9(21)$ & & $13(22)$ & $18(26)$ & & $19(24)$ & $12(23.5)$ & \\
\hline PFI & & & & & & & & & \\
\hline$>12$ months & $39(47)$ & $25(61)$ & $0.6^{\mathrm{e}}$ & $28(50)$ & $36(54)$ & $0.6^{\mathrm{e}}$ & $37(49)$ & $27(56)$ & $0.3^{\mathrm{e}}$ \\
\hline 6-12 months & $21(26)$ & $8(19.5)$ & & $14(25)$ & $15(22)$ & & $15(20)$ & $14(29)$ & \\
\hline$<6$ months & $22(27)$ & $8(19.5)$ & & $14(25)$ & $16(24)$ & & $23(31)$ & $7(15)$ & \\
\hline 5-Year OS & & & & & & & & & \\
\hline Events/person years & $63 / 245$ & $22 / 143$ & & $44 / 172$ & $41 / 216$ & & $56 / 226$ & $29 / 162$ & \\
\hline 5-Year OS (\%) & 24.2 & 41.1 & & 24.4 & 34.4 & & 24.1 & 38.1 & \\
\hline 5-Year PFS & & & & & & & & & \\
\hline Events/person years & $78 / 156$ & $29 / 101$ & & $54 / 108$ & $53 / 149$ & & $68 / 143$ & $39 / 114$ & \\
\hline 5-Year PFS (\%) & 9.2 & 32.6 & & 8.7 & 23.1 & & 13.9 & 21.2 & \\
\hline
\end{tabular}

Values in italics are statistically significant $(P<0.05)$

$P F I$ platinum-free interval, $P F S$ progression-free survival defined as the time interval between date of diagnosis and the date of disease recurrence (pathology report or radiology) or death, whichever occurred first

${ }^{a}$ PD-1 expression in intra-epithelial lymphocytes and PD-L1 expression in intra-epithelial macrophages

${ }^{\mathrm{b}}$ Of the patients who died within 5 years after diagnosis, all but two of 85 died of causes related to HGSC

${ }^{\mathrm{c}} t$ test

${ }^{\mathrm{d}} \mathrm{Chi}^{2}$ test

${ }^{\mathrm{e}}$ Mann-Whitney $U$ test

Detection Kit for the Ventana platform. Placenta and macrophages in tonsil were used as a positive control for PD-L1 (high and low expression, respectively). Macrophages and lymphocytes, and epithelial cells in tonsil, were positive and negative controls, respectively, for CD68, CD163, CD3, and PD-1.

\section{Scoring}

Hematoxylin and eosin, PD-L1, CD68, CD3, PD-1, and CD163 were stained on consecutive sections enabling the evaluation of corresponding tumor areas. We scored lymphocytes located within the tumor epithelium, and only intra- 
Table 2 Summary of antibodies and immunohistochemistry procedures

\begin{tabular}{|c|c|c|c|c|c|c|c|}
\hline Antigen & Clone & Cat. no. & Supplier & Dilution & Platform & Ag retrieval $(\mathrm{pH})$ & $\begin{array}{l}\text { Ab incubation } \\
\left(\mathrm{min} /{ }^{\circ} \mathrm{C} \text { or } \mathrm{RT}\right)\end{array}$ \\
\hline PD-L1 & $22 \mathrm{C} 3$ & M3653 & Agilent Dako & $1: 50$ & Dako Autostainer & DT 1699 (6) & $30 / \mathrm{RT}$ \\
\hline PD-1 & NAT105 & $315 \mathrm{M}$ & Cell Marque (Sigma) & $1: 100$ & Dako Autostainer & DT 1699 (6) & $30 / \mathrm{RT}$ \\
\hline CD68 & PG-M1 & M0876 & Agilent Dako & $1: 100$ & Dako Autostainer & DT 2367 (9) & $30 / \mathrm{RT}$ \\
\hline CD163 & MRQ-26 & $760-4437$ & Ventana & RTU & Ventana Benchmark Ultra & CC1 (8.5) & $32 / 36$ \\
\hline CD3 & Poly & A 0452 & Agilent Dako & $1: 200$ & Dako Autostainer & DT 2367 (9) & $30 / \mathrm{RT}$ \\
\hline \multicolumn{8}{|c|}{ Large sections } \\
\hline PD-L1 & $22 \mathrm{C} 3$ & M365529 & Agilent Dako & $1: 40$ & Ventana Benchmark Ultra & CC1 (8.5) & $64 / 36$ \\
\hline PD-1 & NAT105 & Ab52587 & Abcam & $1: 50$ & Ventana Benchmark Ultra & CC1 (8.5) & $32 / 36$ \\
\hline PD-1 & NAT105 & $315 \mathrm{M}$ & Cell Marque (Sigma) & $1: 100$ & Dako Autostainer & DT 1699 (6) & $30 / \mathrm{RT}$ \\
\hline CD68 & PG-M1 & M0786 & Dako & $1: 100$ & Ventana Benchmark Ultra & $\mathrm{CC} 1(8.5)$ & $32 / 36$ \\
\hline CD3 & $2 \mathrm{GV6}$ & $760-4341$ & Ventana & RTU & Ventana Benchmark Ultra & CC1 (8.5) & $32 / 36$ \\
\hline
\end{tabular}

$R T$ room temperature, $R T U$ ready to use, DT 1699 Dako Target retrieval solution 1699, DT 2367 Dako Target retrieval solution 2367 , CC1 cell conditioning 1

epithelial and luminal macrophages were evaluated. All stained slides, except PD-L1, were evaluated by two investigators (LMF, SWF), and discordant cases were discussed until consensus was achieved. PD-L1 evaluation was performed by a pathologist (SWF) with experience in PD-L1 scoring of lung cancer in the clinical setting. Scoring was performed blinded from clinical data. The macrophage marker CD68 facilitated the distinction between macrophages and cancer cells when evaluating PD-L1 expression (see Fig. 1). We determined the average $(0 \%,<1 \%, 1-4 \%, \geq 5 \%$ for PD-L1 and PD- 1 and $0 \%$, $<1 \%, 1 \%, 2-4 \%, \geq 5 \%$ for CD3, CD68, and CD163) of the total cell amount in each core, excluding areas with stroma, acute inflammation, and necrosis. Examples of score intervals for PD-L1 and PD-1 are presented in Fig. 1. Further, we stained and evaluated 13 cases of paired tissue microarray and whole sections for comparison.

\section{In silico validation of CD274 (PD-L1) and CD3G (CD3) mRNA expression}

An independent public gene expression data set consisting of 285 high-grade serous and endometrioid, borderline as well as
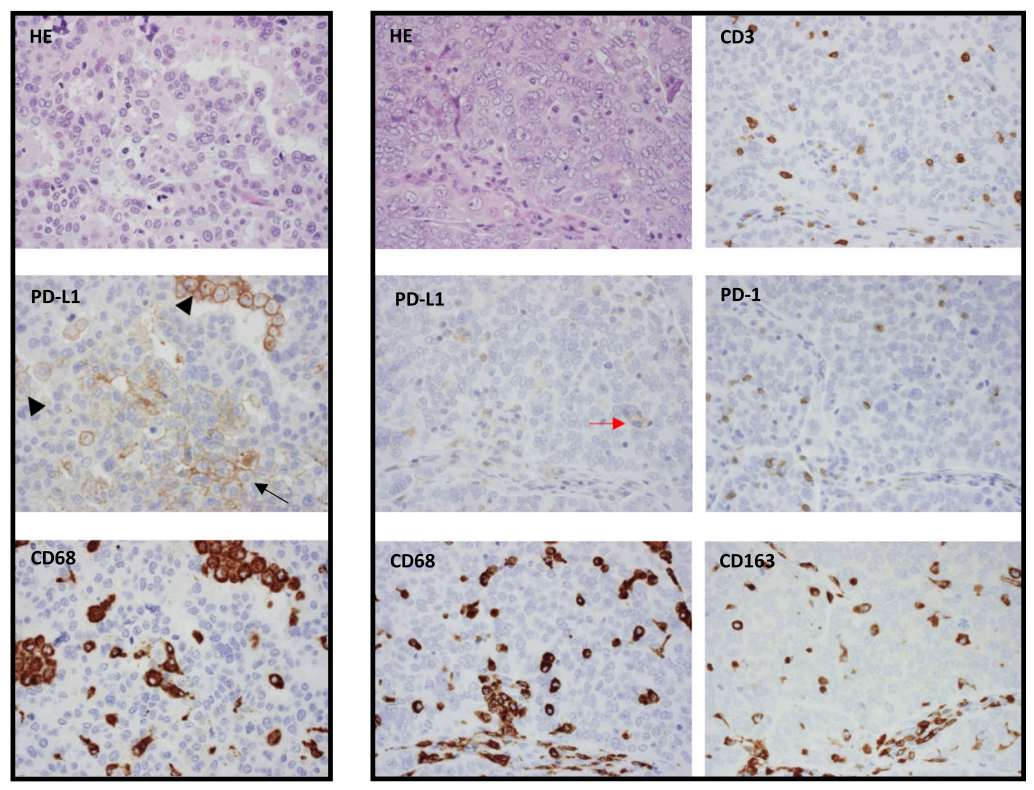

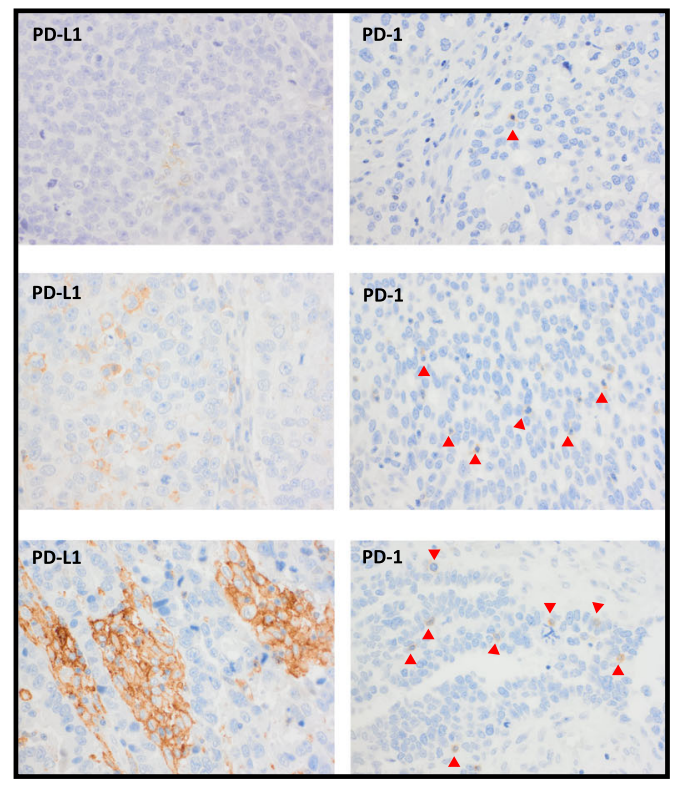

shows a PD-L1 positive macrophage. Stroma seen at the bottom of the pictures excluded from scoring. Examples of areas rich in PD-L1 expression in intra-epithelial macrophages and PD-1 expression in intraepithelial lymphocytes from cores with scores $<1 \%, 1-4 \%$, and $\geq 5 \%$ (from top to bottom) (right panel). The red arrowheads show PD-1 positive lymphocytes. Magnification, $\times 40$ 
low-grade serous and endometrioid ovarian tumors, fallopian tube, and primary peritoneal cancers was downloaded from Gene Expression Omnibus (GSE9891) [14]. We selected 203 cases with high-grade serous histology and studied the relationship between expression of CD274 (probe 227458_at, encoding PD-L1) and CD3G (probe 206804_at, encoding CD3) and 5-year OS. We compared high versus low expression using the median mRNA expression level as cutoff.

\section{Statistical analyses}

The prognostic value was investigated using 5-year OS as endpoint, defined as the time interval between date of diagnosis and death.

Statistical analyses were performed with the Statistical Package for the Social Sciences, Windows version 25. Survival analyses were performed using the Kaplan-Meier method, and differences between groups were tested using the log-rank test. The effect of the expression of the immune markers on OS was expressed using hazard ratios (HRs) with $95 \%$ confidence interval (CI), estimated using univariable and multivariable Cox regression. The multivariable analysis adjusted for clinical factors known to influence HGSC survival, age at diagnosis $(\geq 70$ vs. $<70$ ), stage (IV vs. III), and residual tumor following primary cytoreductive surgery (macroscopic residual tumor vs. no) $[1,26,27]$, were analyzed as binary factors. All $P$ values are two sided. The three patients who underwent bowel obstruction surgery only were considered as having macroscopic residual tumor for analyses.

In the external data set, statistical analyses were performed in $\mathrm{R}$ version 3.3.3. Associations between $C D 3 G$ and $C D 274$ mRNA levels and 5-year OS were assessed using the KaplanMeier method, and HRs with 95\% confidence interval were calculated in univariable analysis using Cox proportional hazard regression ( $\mathrm{R}$ "survival” package version 2.41-3).

\section{Results}

We found significant positive associations between all markers studied, except PD-1 and CD163 (chi $\left.{ }^{2}, P=0.06\right)$. Strong positive associations were observed between PD-L1, PD-1, and CD3 (chi ${ }^{2}, P<0.001$ ); PD-1, CD3, and CD68 (chi ${ }^{2}, P<0.001$ ); and PD-L1, CD3, CD163, and CD68 (chi $\left.{ }^{2}, P<0.01\right)$. Associations between expression of each marker and clinical parameters are shown in Table 1.

\section{Patterns and prognostic effects of PD-1 and PD-L1 expression}

PD-1 was almost exclusively expressed by lymphocytes (Fig. 1). We observed both partial and complete, as well as weak and moderate, membranous staining. Patients with $\geq 50 \%$ cores with PD-1 expression $\geq 1 \%$ (39/130), considered to have high expression, had longer OS $(P=0.007$; Fig. 2a and Table 3), even when adjusting for well-known prognostic factors (Table 4). Interestingly, high PD-1 expression was associated with a stronger survival benefit when the analysis was restricted to the 43 cases with high $\mathrm{CD} 3$ expression (31 cases PD-1 high vs. 12 cases PD-1 low, HR 0.33 [0.14-0.77], $P=0.01)$.

PD-L1 was expressed mainly by macrophages, and to a far lesser extent by tumor cells and lymphocytes (Fig. 1). We evaluated the membranous expression that was predominantly partial and weak, but also observed granular cytoplasmic expression. Using the same cutoff as for PD- $1(\geq 50 \%$ cores with $\geq 1 \%$ PD-L1 expressing macrophages), we identified only 15 / 130 patients as positive. The survival benefit of this small group was high and statistically significant but uncertain (HR 0.20 [0.07-0.66], $P=0.007$ ). Thus, we considered cases with $\geq 2$ cores with $\geq 1 \%$ PD-L1 expressing macrophages as positive (26/130) and found a significant association with improved OS also within this group $(P=0.02$; Fig. $2 \mathrm{~b}$ and
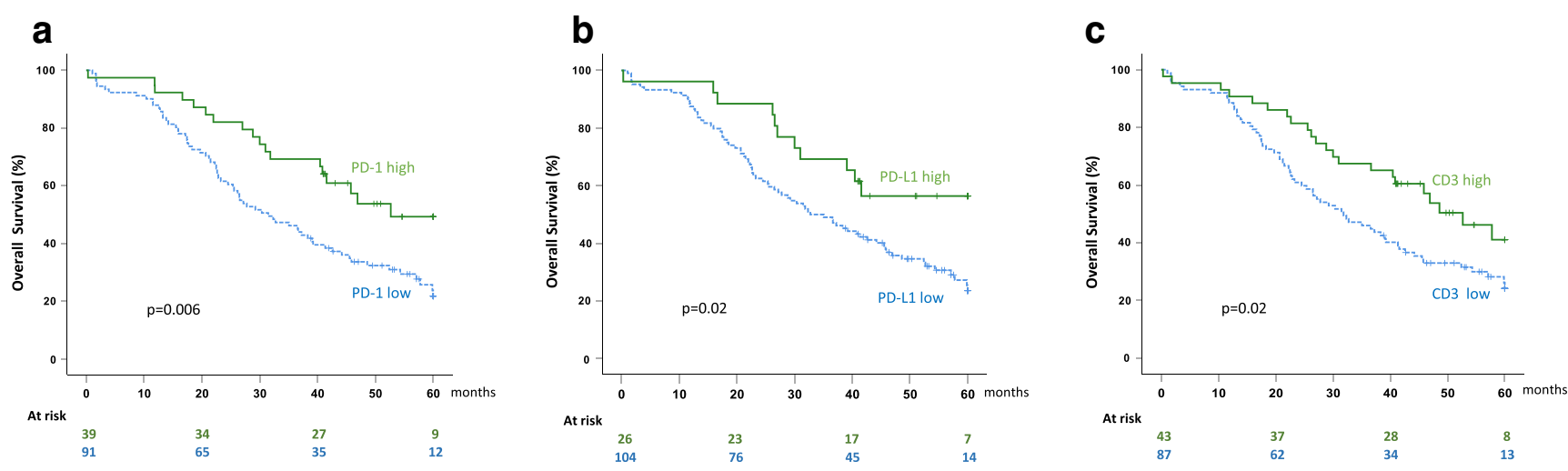

Fig. 2 Association between OS and the expression of PD-1 in lymphocytes, PD-L1 in macrophages, and CD3 expression, within the tumor epithelium. a Patients with high PD-1 expression had longer survival compared with patients with low PD-1 expression. b Patients with high
PD-L1 expression had longer survival compared with patients with low PD-L1 expression. c Patients with high CD3 expression had longer survival compared with patients with low $\mathrm{CD} 3$ expression. $P$ values were calculated using the log-rank test 
Table 3 Univariable analyses of overall survival

\begin{tabular}{lllll}
\hline & \multicolumn{4}{l}{ 5-Year OS univariable Cox } \\
\cline { 3 - 5 } & & $n$ (events) & HR (95\% CI $)$ & $P$ \\
\hline CD3 $^{\text {a }}$ & Low & $87(63)$ & 1 & \\
& High & $43(22)$ & $0.58(0.35-0.94)$ & 0.03 \\
PD-1 $^{\text {a }}$ & Low & $91(67)$ & 1 & \\
& High & $39(18)$ & $0.49(0.29-0.82)$ & 0.007 \\
PD-L1 $^{\text {a }}$ & Low & $104(74)$ & 1 & \\
& High & $26(11)$ & $0.47(0.25-0.89)$ & 0.02 \\
Age at diagnosis & $<70$ & $85(47)$ & 1 & \\
& $\geq 70$ & $45(38)$ & $2.5(1.6-3.8)$ & $<0.001$ \\
Stage & III & $99(57)$ & 1 & \\
& IV & $31(28)$ & $2.9(1.8-4.5)$ & $<0.001$ \\
Residual tumor & No & $75(42)$ & 1 & \multirow{2}{*}{0.001} \\
& Yes & $55(43)$ & $2.0(1.3-3.1)$ & 0.001 \\
\hline
\end{tabular}

Values in italics are statistically significant $(P<0.05)$

${ }^{a}$ Intra-epithelial CD3 expression, PD-1 expression in intra-epithelial lymphocytes, and PD-L1 expression in intra-epithelial macrophages

Table 3). Because of scarce PD-L1 expression in tumor cells and lymphocytes, no relevant cutoff could be determined, precluding further analyses.

Furthermore, we explored the survival benefit of the subgroup with higher expression of both PD-1 and PD-L1 and found lower hazards of death compared with each marker alone (19 cases PD-1/PD-L1 high vs. 84 cases PD-1/PD-L1 low, HR 0.36 [0.17-0.79], $P=0.01$, Supplementary Table 1).

Table 4 Multivariable analyses of overall survival

\begin{tabular}{llll}
\hline & & \multicolumn{2}{l}{ 5-Year OS multivariable Cox } \\
\cline { 3 - 4 } & & HR $(95 \%$ CI $)$ & $P$ \\
\hline CD3 $^{\text {a }}$ & High vs. low & $0.60(0.37-0.98)$ & 0.04 \\
Age at diagnosis & $\geq 70$ vs. $<70$ & $2.3(1.5-3.6)$ & $<0.001$ \\
Stage & IV vs. III & $2.8(1.8-4.5)$ & $<0.001$ \\
Residual tumor & Yes vs. no & $1.8(1.2-2.7)$ & 0.01 \\
PD-1 $^{\text {a }}$ & High vs. low & $0.55(0.32-0.94)$ & 0.03 \\
Age at diagnosis & $\geq 70$ vs. $<70$ & $2.3(1.5-3.5)$ & $<0.001$ \\
Stage & IV vs. III & $2.6(1.6-4.2)$ & $<0.001$ \\
Residual tumor & Yes vs. no & $1.8(1.2-2.8)$ & 0.008 \\
PD-L1 $^{\text {a }}$ & High vs. low & $0.62(0.32-1.2)$ & 0.1 \\
Age at diagnosis & $\geq 70$ vs. $<70$ & $2.3(1.5-3.6)$ & $<0.001$ \\
Stage & IV vs. III & $2.8(1.5-3.6)$ & $<0.001$ \\
Residual tumor & Yes vs. no & $1.7(1.1-2.6)$ & 0.02 \\
\hline
\end{tabular}

Multivariable analysis of each immune marker including well-known prognostic factors above. Values in italics are statistically significant $(P<0.05)$

${ }^{a}$ Intra-epithelial CD3 expression, PD-1 expression in intra-epithelial lymphocytes, and PD-L1 expression in intra-epithelial macrophages

\section{Lymphocytes and macrophages in HGSC}

Patients with $\geq 50 \%$ cores with $\geq 2 \%$ lymphocytes (CD3 high, 43/130) had longer OS compared with patients with lower expression ( $P=0.03$; Fig. 2c and Table 3 ), even when adjusting for well-known prognostic factors $(P=0.04$; Table 4$)$.

No significant OS difference between patients with $\geq 50 \%$ cores with $\geq 2 \%$ macrophages (CD68 high, 70/130) and patients with lower CD68 expression (60/130) was observed (HR 0.74 [0.48-1.1], $P=0.2$ ). Furthermore, we did not find any difference in OS between patients with $\geq 50 \%$ cores with CD163 expression $\geq 2 \%$ (CD163 high, 51/130) and patients with lower CD163 expression (79/130, HR 0.70 [0.44-1.1], $P=0.1)$. However, in the subgroup of patients with no macroscopic residual tumor, higher CD163 expression was associated with better outcome $(P=0.02$, Supplementary Table 2).

\section{Whole tissue sections}

We observed concordance between evaluations performed on tissue microarray and whole tissue sections in the majority of the 17 pairs investigated (13 cases, four of which with paired samples from adnexa and metastatic site). Comparing the average staining of the cores and whole tissue sections, the highest concordance was observed for PD-1, where only 1/17 pairs was discordant. Regarding PD-L1 in macrophages, scoring on tissue microarray underestimated whole tissue section scoring in $3 / 17$ pairs and for $\mathrm{CD} 3$, and 2/17 pairs were discordant.

Of note, tissue microarray and whole section immunostainings were performed on the Dako Autostainer and Ventana system, respectively, which did not seem to affect the performance of the antibodies. PD-1 immunostaining on whole sections was discordant on the Ventana system, and therefore, we performed the comparison on the same platform as the tissue microarray, the Dako Autostainer.

\section{High CD274 mRNA expression linked with survival benefit}

In the external data set, high expression of CD274 (encoding PD-L1) was associated with improved OS among HGSC patients ( $P=0.03$; Supplementary Fig. 2). However, no significant associations between $C D 3 G$ (encoding CD3) and OS were found.

\section{Discussion}

In this study, we found that PD-L1 was expressed mainly by macrophages, which has previously been reported [19, 28, 29] and not by tumor cells as some previous studies suggest [17, 18, 20, 30]. Remarkably, macrophage staining, which was necessary for the correct mapping of PD-L1 expression in 
macrophages vs. tumor cells and revealed the predominance of expression on macrophages, was not performed in the previous studies where PD-L1 expression in macrophages was not described. Moreover, the specificity and sensitivity of antiPD-L1 antibodies have been debated, and based on results from The Blueprint Project [31] and Brunnström et al. [32], we decided to use the FDA-approved $22 \mathrm{C} 3$ clone in the present study.

In our cohort of 130 consecutive cases of advanced HGSC, higher expression of intra-epithelial lymphocytes (CD3), PD1 (in lymphocytes), and PD-L1 (in macrophages) was a predictor of better outcome with similar hazards of death, even after adjusting for age at diagnosis, stage, and residual tumor after primary surgery. In agreement with our results, previous studies have reported the significantly decreased risk of death in patients with higher intra-epithelial CD3 expression (HR 0.50 [0.36-0.69] and HR 0.45 [0.34-0.58] in two different meta-analyses) $[12,13]$. Regarding PD-1, two previous studies have showed a survival benefit for patients with ovarian carcinoma whose tumors express PD-1 [18, 33]. However, in one of the studies, PD-1 expression was described in tumor cells in a majority of the cases (151/172) in addition to lymphocytes [18]. In our study, we did not observe any PD-1 staining in tumor cells, consistent with one other previous report [33]. In fact, the study reporting PD-1 expression in tumor cells used different PD-1 antibodies to stain tissue microarray and whole sections, and importantly, no PD-1positive cancer cells were observed in whole sections.

Higher PD-L1 expression predicts inferior survival in several cancer forms [34], supporting the theory of tumor cells upregulating PD-L1 in order to suppress $\mathrm{T}$ cells, thereby promoting tumor growth. In contrast, previous studies in HGSC, in addition to our cohort and in silico validation presented herein, have shown a survival benefit of high PD-L1 expression in macrophages [19, 28, 29]. Interestingly, high PD-1 expression was associated with an even stronger survival benefit when the analysis was restricted to cases with high CD3 expression. Thus, despite the PD-1/PD-L1 pathway being a negative regulator of $T$ cell activation, women which tumors had a higher PD-1 expression in intra-epithelial lymphocytes and a higher PD-L1 expression in intra-epithelial macrophages had longer survival. Indeed, a previous study referred to adaptive immune resistance, wherein activated $\mathrm{T}$ cells may trigger negative feedback mechanisms, resulting in an immunological stalemate [19]. The sparse expression of PD-L1 in HGSC tumor cells, in contrast to other malignancies [31], remains an unresolved issue. Nevertheless, the observed survival benefit suggests that the potential of tumor immunity may be harnessed in subsets of HGSC.

We did not observe a prognostic value of intra-epithelial macrophages (CD68) in the present study. Only two previous studies - smaller and including mixed histological typeswere found, making it difficult to compare with our study $[35,36]$. Similarly, some smaller studies with mixed histological types, evaluating CD163 expressing macrophages in ovarian carcinoma, have been published. Apparently, the results of a previous study that showed a survival benefit for patients whose tumors displayed low CD163 expression in multivariable analysis [37] are in conflict with our results. However, in this study, peritumoral stroma and not intraepithelial CD163 expression were evaluated. Another study did not report any difference in prognosis between groups with high vs. low intra-epithelial CD163 expression [38], which is in agreement with our results. However, higher CD163 expression was associated with better outcome in the subgroup of patients with no macroscopic tumor after surgery in our cohort. This result is apparently in conflict with the theory that M2 macrophages promote tumor progression, thereby negatively affecting survival [39]. Of note, the macrophage classification in classical versus alternative activated represents a simplification of the heterogeneous macrophage population in tumors [39], and a more detailed characterization of macrophages in HGSC may be required.

Although some previous studies have investigated the prognostic value of PD-L1 and PD-1 in HGSC, we found ambiguous issues that we addressed in the present study. According to our results, the main cells expressing PD-L1 are macrophages (and not tumor cells) and that PD-1 is almost exclusively expressed by lymphocytes (and not by tumor cells). Most previous studies include smaller cohorts and, in some cases, not consecutively collected cohorts with a selected material concerning residual disease [19] or stage [30]. Further, this is the first study reporting on the prognostic value of CD68 and CD163 in a pure advanced HGSC cohort, as previous smaller studies included mixed histologies which differ greatly in clinical presentation and prognosis. Other strengths of this study include the use of a contemporary and comprehensive tissue microarray, as well as validation using tissue whole sections. Given the number of cores per case and the fact that the tissue microarray includes cores from both primary and metastatic sites, our tissue microarray outperforms previous ovarian carcinoma tissue microarray cohorts. Furthermore, the PD-L1 scoring was performed by a pathologist with experience from PD-L1 scoring of lung cancer in the clinical setting.

Some of the limitations include that only 25 patients were tested for BRCA1/2 mutations in our cohort, precluding the possibility of performing potentially interesting survival analyses in these subgroups. However and interestingly, we noted a strong positive correlation between the expression of the immune marker that best predicted prognosis, PD-1, and platinum sensitivity. The group of platinum sensitive tumors is enriched with tumors showing homologous recombination deficiency [40], and this may imply a relationship between tumor immunogenicity and homologous recombination deficiency, as recently suggested [10].

In conclusion, we corroborate that PD-L1 is primarily expressed by macrophages and found that higher expression 
of lymphocytes, PD-1 in lymphocytes, and PD-L1 in macrophages within the tumor epithelium confers a significant survival advantage in advanced HGSC.

Acknowledgments The authors would like to acknowledge Stina Nordström for collection of clinical data and Anna Ebbesson for assistance with TMAs and stainings.

Author contribution All authors of this research paper have participated in the planning, execution, and/or analysis of the study. LMF has collected clinical data, evaluated the TMA, performed the statistical analyses and interpretation of the clinical data, and is the main writer. SWF is responsible for the TMA construction and evaluation of TMA and whole sections. SM supervised the collection of clinical data. NSA downloaded data and performed analyses in the validation cohort. LH revised statistics. The rest of authors contributed as senior expertise. All authors of this paper have critically revised the intellectual content and approved the final version submitted.

Funding information Open access funding provided by Lund University. IH received grant support from the Swedish Cancer Society (2015/486), the G Nilsson Cancer Foundation (2017/728), the B Kamprad Foundation (FBKS 2017/22), the Cancer and Allergy Foundation (2017/150428), King Gustaf V's Jubilee Foundation (2017/174161), the Lund University Hospital Research Foundation (2017-033), and governmental funding of clinical research within the National Health Services (ALF) (2014/10601). LMF received governmental funding of clinical research within the National Health Services. SWF received grant support from BioCare (governmental funding).

\section{Compliance with ethical standards}

Ethical approval for this study was granted by the Ethics Committee at Lund University, Sweden, waiving the requirement for informed consent.

Conflict of interest Dr. Malander has received honoraria from AstraZeneca and Tesaro for advisory board participation during the conduct of this study. Dr. Martin de la Fuente received grant support from Roche in collaboration with the Swedish Society of Gynecological Oncology. The rest of the authors declare that they have no conflicts of interest to report.

Open Access This article is licensed under a Creative Commons Attribution 4.0 International License, which permits use, sharing, adaptation, distribution and reproduction in any medium or format, as long as you give appropriate credit to the original author(s) and the source, provide a link to the Creative Commons licence, and indicate if changes were made. The images or other third party material in this article are included in the article's Creative Commons licence, unless indicated otherwise in a credit line to the material. If material is not included in the article's Creative Commons licence and your intended use is not permitted by statutory regulation or exceeds the permitted use, you will need to obtain permission directly from the copyright holder. To view a copy of this licence, visit http://creativecommons.org/licenses/by/4.0/.

\section{References}

1. du Bois A, Reuss A, Pujade-Lauraine E, Harter P, Ray-Coquard I, Pfisterer J (2009) Role of surgical outcome as prognostic factor in advanced epithelial ovarian cancer: a combined exploratory analysis of 3 prospectively randomized phase 3 multicenter trials: by the Arbeitsgemeinschaft Gynaekologische Onkologie Studiengruppe Ovarialkarzinom (AGO-OVAR) and the Groupe d'Investigateurs Nationaux pour les etudes des cancers de l'Ovaire (GINECO). Cancer 115(6):1234-1244

2. Siegel RL, Miller KD, Jemal A (2017) Cancer statistics, 2017. CA Cancer J Clin 67(1):7-30

3. Moore K, Colombo N, Scambia G, Kim BG, Oaknin A, Friedlander M, Lisyanskaya A, Floquet A, Leary A, Sonke GS, Gourley C, Banerjee S, Oza A, González-Martín A, Aghajanian C, Bradley W, Mathews C, Liu J, Lowe ES, Bloomfield R, DiSilvestro P (2018) Maintenance olaparib in patients with newly diagnosed advanced ovarian cancer. N Engl J Med 379(26):2495-2505

4. Robert C, Long GV, Brady B, Dutriaux C, Maio M, Mortier L, Hassel JC, Rutkowski P, McNeil C, Kalinka-Warzocha E, Savage KJ, Hernberg MM, Lebbé C, Charles J, Mihalcioiu C, ChiarionSileni V, Mauch C, Cognetti F, Arance A, Schmidt H, Schadendorf D, Gogas H, Lundgren-Eriksson L, Horak C, Sharkey B, Waxman IM, Atkinson V, Ascierto PA (2015) Nivolumab in previously untreated melanoma without BRAF mutation. N Engl J Med 372(4): 320-330

5. Brahmer J, Reckamp KL, Baas P, Crinò L, Eberhardt WE, Poddubskaya E, Antonia S, Pluzanski A, Vokes EE, Holgado E, Waterhouse D, Ready N, Gainor J, Arén Frontera O, Havel L, Steins M, Garassino MC, Aerts JG, Domine M, Paz-Ares L, Reck M, Baudelet C, Harbison CT, Lestini B, Spigel DR (2015) Nivolumab versus docetaxel in advanced squamous-cell nonsmall-cell lung cancer. N Engl J Med 373(2):123-135

6. Bellmunt $J$ et al (2017) Pembrolizumab as second-line therapy for advanced urothelial carcinoma. N Engl J Med 376(11):1015-1026

7. Hamanishi J et al (2015) Safety and antitumor activity of anti-PD-1 antibody, nivolumab, in patients with platinum-resistant ovarian cancer. J Clin Oncol 33(34):4015-4022

8. Hamanishi J, Mandai M, Konishi I (2016) Immune checkpoint inhibition in ovarian cancer. Int Immunol 28(7):339-348

9. Wang $Z$ et al (2019) Niraparib activates interferon signaling and potentiates anti-PD-1 antibody efficacy in tumor models. Sci Rep 9(1): 1853

10. Strickland KC, Howitt BE, Shukla SA, Rodig S, Ritterhouse LL, Liu JF, Garber JE, Chowdhury D, Wu CJ, D'Andrea AD, Matulonis UA, Konstantinopoulos PA (2016) Association and prognostic significance of BRCA1/2-mutation status with neoantigen load, number of tumor-infiltrating lymphocytes and expression of PD-1/PDL1 in high grade serous ovarian cancer. Oncotarget 7(12):1358713598

11. Gibney GT, Weiner LM, Atkins MB (2016) Predictive biomarkers for checkpoint inhibitor-based immunotherapy. Lancet Oncol 17(12):e542-e551

12. Li J, Wang J, Chen R, Bai Y, Lu X (2017) The prognostic value of tumor-infiltrating $\mathrm{T}$ lymphocytes in ovarian cancer. Oncotarget 8(9):15621-15631

13. Hwang WT, Adams SF, Tahirovic E, Hagemann IS, Coukos G (2012) Prognostic significance of tumor-infiltrating T cells in ovarian cancer: a meta-analysis. Gynecol Oncol 124(2):192-198

14. Tothill RW et al (2008) Novel molecular subtypes of serous and endometrioid ovarian cancer linked to clinical outcome. Clin Cancer Res 14(16):5198-5208

15. Cancer Genome Atlas Research, N (2011) Integrated genomic analyses of ovarian carcinoma. Nature 474(7353):609-615

16. Verhaak RG, Tamayo P, Yang JY, Hubbard D, Zhang H, Creighton CJ, Fereday S, Lawrence M, Carter SL, Mermel CH, Kostic AD, Etemadmoghadam D, Saksena G, Cibulskis K, Duraisamy S, Levanon K, Sougnez C, Tsherniak A, Gomez S, Onofrio R, Gabriel S, Chin L, Zhang N, Spellman PT, Zhang Y, Akbani R, Hoadley KA, Kahn A, Köbel M, Huntsman D, Soslow RA, Defazio A, Birrer MJ, Gray JW, Weinstein JN, Bowtell DD, Drapkin R, 
Mesirov JP, Getz G, Levine DA, Meyerson M, Cancer Genome Atlas Research Network (2013) Prognostically relevant gene signatures of high-grade serous ovarian carcinoma. J Clin Invest 123(1): $517-525$

17. Hamanishi $J$ et al (2007) Programmed cell death 1 ligand 1 and tumor-infiltrating CD8+ T lymphocytes are prognostic factors of human ovarian cancer. Proc Natl Acad Sci U S A 104(9):33603365

18. Darb-Esfahani S, Kunze CA, Kulbe H, Sehouli J, Wienert S, Lindner J, Budczies J, Bockmayr M, Dietel M, Denkert C, Braicu I, Jöhrens K (2016) Prognostic impact of programmed cell death-1 (PD-1) and PD-ligand 1 (PD-L1) expression in cancer cells and tumor-infiltrating lymphocytes in ovarian high grade serous carcinoma. Oncotarget 7(2):1486-1499

19. Webb JR, Milne K, Kroeger DR, Nelson BH (2016) PD-L1 expression is associated with tumor-infiltrating T cells and favorable prognosis in high-grade serous ovarian cancer. Gynecol Oncol 141(2): 293-302

20. Wang Q, Lou W, di W, Wu X (2017) Prognostic value of tumor PDL1 expression combined with CD8(+) tumor infiltrating lymphocytes in high grade serous ovarian cancer. Int Immunopharmacol 52:7-14

21. Yuan X, Zhang J, Li D, Mao Y, Mo F, du W, Ma X (2017) Prognostic significance of tumor-associated macrophages in ovarian cancer: a meta-analysis. Gynecol Oncol 147(1):181-187

22. Altman DG, McShane L, Sauerbrei W, Taube SE (2012) Reporting Recommendations for Tumor Marker Prognostic Studies (REMARK): explanation and elaboration. PLoS Med 9(5): e1001216

23. Kurman, R.J., Carcangiu, M.L., Herrington, C.S., Young, R.H., Tumors of the ovary, in WHO Classification of Tumours of Female Reproductive Organs R.J. Kurman, Carcangiu, M.L., Herrington, C.S., Young, R.H., Editor. 2014

24. Prat J, F.C.o.G (2014) Oncology, Staging classification for cancer of the ovary, fallopian tube, and peritoneum. Int J Gynaecol Obstet 124(1):1-5

25. Stuart GC, Kitchener H, Bacon M, duBois A, Friedlander M, Ledermann J, Marth C, Thigpen T, Trimble E, participants of 4th Ovarian Cancer Consensus Conference (OCCC), Gynecologic Cancer Intergroup (2011) 2010 Gynecologic Cancer InterGroup (GCIG) consensus statement on clinical trials in ovarian cancer: report from the Fourth Ovarian Cancer Consensus Conference. Int J Gynecol Cancer 21(4):750-755

26. Sabatier R, Calderon B Jr, Lambaudie E, Chereau E, Provansal M, Cappiello MA, Viens P, Rousseau F (2015) Prognostic factors for ovarian epithelial cancer in the elderly: a case-control study. Int $\mathrm{J}$ Gynecol Cancer 25(5):815-822

27. Heintz AP et al (2006) Carcinoma of the ovary. FIGO 26th Annual Report on the Results of Treatment in Gynecological Cancer. Int $J$ Gynaecol Obstet 95(Suppl 1):S161-S192

28. Gottlieb CE, Mills AM, Cross JV, Ring KL (2017) Tumorassociated macrophage expression of PD-L1 in implants of high grade serous ovarian carcinoma: a comparison of matched primary and metastatic tumors. Gynecol Oncol 144(3):607-612
29. Lo CS, Sanii S, Kroeger DR, Milne K, Talhouk A, Chiu DS, Rahimi K, Shaw PA, Clarke BA, Nelson BH (2017) Neoadjuvant chemotherapy of ovarian cancer results in three patterns of tumorinfiltrating lymphocyte response with distinct implications for immunotherapy. Clin Cancer Res 23(4):925-934

30. Fucikova J et al (2019) TIM-3 dictates functional orientation of the immune infiltrate in ovarian cancer. Clin Cancer Res

31. Hirsch FR, McElhinny A, Stanforth D, Ranger-Moore J, Jansson M, Kulangara K, Richardson W, Towne P, Hanks D, Vennapusa B, Mistry A, Kalamegham R, Averbuch S, Novotny J, Rubin E, Emancipator K, McCaffery I, Williams JA, Walker J, Longshore J, Tsao MS, Kerr KM (2017) PD-L1 immunohistochemistry assays for lung cancer: results from phase 1 of the blueprint PD-L1 IHC assay comparison project. J Thorac Oncol 12(2):208-222

32. Brunnstrom $\mathrm{H}$ et al (2017) PD-L1 immunohistochemistry in clinical diagnostics of lung cancer: inter-pathologist variability is higher than assay variability. Mod Pathol 30(10):1411-1421

33. Webb JR, Milne K, Nelson BH (2015) PD-1 and CD103 are widely coexpressed on prognostically favorable intraepithelial CD8 T cells in human ovarian cancer. Cancer Immunol Res 3(8):926-935

34. Wang Q, Liu F, Liu L (2017) Prognostic significance of PD-L1 in solid tumor: an updated meta-analysis. Medicine (Baltimore) 96(18):e6369

35. Tanaka Y, Kobayashi H, Suzuki M, Kanayama N, Suzuki M, Terao $\mathrm{T}$ (2004) Upregulation of bikunin in tumor-infiltrating macrophages as a factor of favorable prognosis in ovarian cancer. Gynecol Oncol 94(3):725-734

36. Wan T, Liu JH, Zheng LM, Cai MY, Ding T (2009) Prognostic significance of tumor-associated macrophage infiltration in advanced epithelial ovarian carcinoma. Ai Zheng 28(3):323-327

37. Lan C, Huang X, Lin S, Huang H, Cai Q, Wan T, Lu J, Liu J (2013) Expression of M2-polarized macrophages is associated with poor prognosis for advanced epithelial ovarian cancer. Technol Cancer Res Treat 12(3):259-267

38. Zhang QW, Liu L, Gong CY, Shi HS, Zeng YH, Wang XZ, Zhao YW, Wei YQ (2012) Prognostic significance of tumor-associated macrophages in solid tumor: a meta-analysis of the literature. PLoS One 7(12):e50946

39. Yang M, McKay D, Pollard JW, Lewis CE (2018) Diverse functions of macrophages in different tumor microenvironments. Cancer Res 78(19):5492-5503

40. Tumiati M, Hietanen S, Hynninen J, Pietilä E, Färkkilä A, Kaipio K, Roering P, Huhtinen K, Alkodsi A, Li Y, Lehtonen R, Erkan EP, Tuominen MM, Lehti K, Hautaniemi SK, Vähärautio A, Grénman S, Carpén O, Kauppi L (2018) A functional homologous recombination assay predicts primary chemotherapy response and longterm survival in ovarian cancer patients. Clin Cancer Res 24(18): $4482-4493$

Publisher's note Springer Nature remains neutral with regard to jurisdictional claims in published maps and institutional affiliations. 